\title{
Miniabrigos meteorológicos: comparação e análise estatística para avaliação de eficiência
}

\author{
Sérgio Serafini Júnior \\ Rogério Rozolen Alves \\ Depto. Geografia FFLCH/USP \\ p. $198-210$
}

\section{revista}

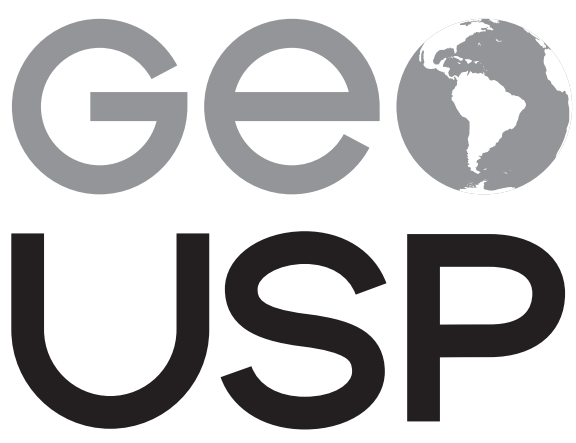

espaço e tempo

Volume 18, no 1 (2014)
Artigo disponível em:

http://www.revistas.usp.br/geousp/article/view/81099

Como citar este ensaio:

SERAFINI JÚNIOR, S.; ALVES, R. R. Miniabrigos meteorológicos: comparação e análise estatística para avaliação de eficiência. GEOUSP - Espaço e Tempo (Online), São Paulo, v. 18, n. 1, p. 198-210, 2014.

\section{$(\mathrm{cc}) \overline{\mathrm{EY}}$}

Este artigo está licenciado sob a Creative Commons Attribution 3.0 License. 


\section{Miniabrigos meteorológicos: comparação e análise estatística para avaliação de eficiência}

Sérgio Serafini Júnior

Rogério Rozolen Alves

\section{Resumo}

No contexto das disciplinas de climatologia do Departamento de Geografia da Universidade de São Paulo, os trabalhos de campo permitem que os alunos pratiquem a análise climatológica usando sensores eletrônicos instalados em miniabrigos meteorológicos, projetados por professores e alunos da pós-graduação.

A partir dos modelos de miniabrigo denominados Tarifa (TAR), Abrigo de Baixo Custo (ABC) e Quatro Águas (4AS), este trabalho faz uma análise comparativa dos dados registrados tomando como referência a Estação Meteorológica Automática (EMA) instalada nas dependências do prédio da Geografia.

Os dados de eficiência desses miniabrigos mostraram que, embora todos tenham apresentado diferenças de resultados em relação à EMA, também tiveram um desempenho bastante satisfatório em $80 \%$ de todo o período amostrado, possibilitando a obtenção de bons resultados para o uso em trabalhos acadêmicos e técnicos.

Palavras-chave: Miniabrigo meteorológico. Climatologia. Biogeografia. Microclima. Geografia.

\section{Mini-weather shelters: statistical analysis for comparison and evaluation of efficiency}

\footnotetext{
Abstract

In the context of the disciplines of climatology of the Department of Geography of University of Sao Paulo, site investigations allow students to practice climatologic analysis using electronic sensors installed in meteorological mini-shelters, designed by tutors and post graduate students.
} 
Based on the models of meteorological mini-shelters described as Tarifa (TAR) [Rate], Abrigo de Baixo Custo (ABC) [Shelter of Low Cost] e Quatro Águas (4AS) [Four Waters], this research made a comparative analysis of registered data, using as reference the Automatic Meteorological Station installed on the premises of the Geography building.

The efficiency data from these mini-shelters demonstrated that, although all presented different results in relation to the Automatic Meteorological Station (EMA), they also presented a performance very satisfactory around $80 \%$ of all sampled period, allowing the achievement of good results for use in academic and technical work.

Keywords: Mini-shelters meteorological. Climatology. Biogeography. Microclimate, Geography.

\section{Introdução}

De modo geral, os trabalhos de campo que acontecem durante o curso de geografia do Departamento de Geografia (DG) da Universidade de São Paulo (USP), visam a capacitação profissional do aluno para a análise adequada do espaço geográfico e suas particularidades.

Basicamente, nos trabalhos de biogeografia e climatologia, dados de temperatura e de umidade relativa do ar são utilizados como indicativos das características gerais da área de estudo, como por exemplo, sobre o período sazonal, a localização latitudinal, a proximidade com o mar, ou mesmo os tipos de uso e ocupação do solo ali predominantes.

Atualmente, tais dados são obtidos através de termo-higrômetros eletrônicos, instalados em miniabrigos meteorológicos projetados e montados por professores e alunos da pós-graduação de climatologia, vinculados ao Laboratório de Climatologia e Biogeografia (LCB).

Esses miniabrigos e termo-higrômetros eletrônicos são utilizados em diferentes disciplinas, consultorias técnicas dadas pelo próprio Departamento de Geografia (DG) ${ }^{1}$ e também por alunos da pós-graduação, como ocorreu nas pesquisas de campo no Núcleo Cunha do Parque Estadual da Serra do Mar (Armani, 2009), no Parque Estadual Veredas do Peruaçu (Serafini Júnior, 2010) e no Parque Nacional Cavernas do Peruaçu (Ibama, 2005).

Constatado o uso frequente desses miniabrigos, este artigo faz uma análise comparativa dos três modelos utilizados pelo Departamento de Geografia; são eles: o miniabrigo Tarifa (TAR), o miniabrigo de Baixo Custo (ABC) e o miniabrigo Quatro Águas (4AS), com o objetivo de verificar a eficiência de cada um deles, em relação ao parâmetro de temperatura.

\section{Os miniabrigos considerados}

Os miniabrigos analisados são produtos de um trabalho constante dos alunos e professores de climatologia em busca de melhorias necessárias para o desenvolvimento do ensino desta disciplina e profissional desenvolvidos fora da universidade.

1 Parque Estadual de Intervales e do trecho sul do rodoanel Mario Covas (SP-21). 
Até o final da década de 90, o DG dispunha de um único modelo adotado pelo Prof. Dr. José Roberto Tarifa, para ser utilizado em trabalhos de campo com alunos da graduação e da pós-graduação. Esse modelo de miniabrigo (modelo TAR)2 é uma caixa de madeira de três faces fechadas e uma aberta, composta por uma cobertura de duas águas e sua instalação é feita por uma haste de madeira para que fique fixado adequadamente na área de estudo.

Seu espaço interno é utilizado para a instalação de instrumentos analógicos; geralmente dois termômetros para cálculo da umidade relativa, além de termômetros de mínima e máxima (Figura 1).

\section{Figura 1}

\section{Miniabrigo TAR, usado em trabalho de campo para o plano de manejo do Par- que Nacional Cavernas do Peruaçu (MG), em 2002}

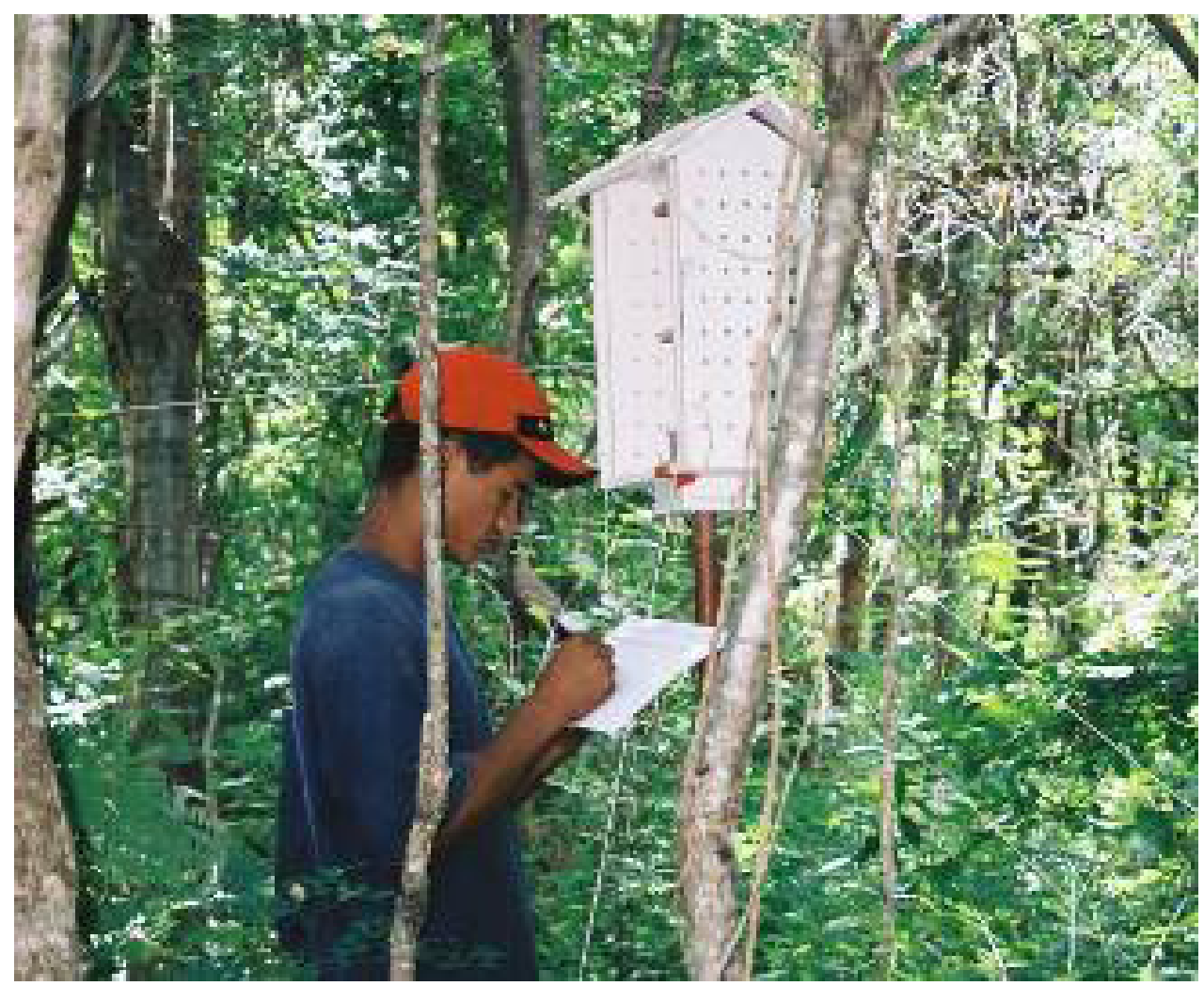

Já no final da década de 90, algumas pesquisas acadêmicas da pós-graduação começaram a ser realizadas com o uso de termo-higrômetros eletrônicos que também eram instalados nos miniabrigos do modelo TAR, resultando em análises mais precisas e mais detalhadas dos objetos de estudos.

Posteriormente, Azevedo e Tarifa (2001) apresentaram um novo modelo denominado de Miniabrigo Meteorológico Aspirado (MMA), projetado para termo-higrômetros eletrônicos e que apresentaram melhor eficiência, tanto no desempenho, como no uso e no custo-benefício. ${ }^{3}$

2 Na etapa de levantamento bibliográfico, não foi encontrado nenhum nome desse miniabrigo. Portanto, neste trabalho, ele é chamado TAR, em referência ao Prof. Dr. José Roberto Tarifa, que o trouxe ao Departamento de Geografia na década de 1990, para ser usado nos trabalhos de campo com seus alunos.

3 O Miniabrigo Meteorológico Aspirado (MMA) não foi comparado aos demais aqui considerados para análise proposta. 
A partir da aquisição de mais termo-higrômetros pelo DG, houve um consequente aumento da frequência do uso desses instrumentos, quando Armani e Galvani (2006) também desenvolveram e avaliaram um novo modelo de miniabrigo, nomeado Abrigo de Baixo Custo (ABC), demonstrando um bom desempenho, além de ter um custo reduzido para sua fabricação.

Basicamente, o miniabrigo ABC tem seu formato cônico. É composto por chapas sobrepostas de latão galvanizado, formando uma veneziana circular contínua que favorece a ventilação passiva no seu interior, onde fica alojado o termo-higrômetro eletrônico. Sua fixação se dá através de uma haste de metal (Figura 2).

\section{Figura 2}

\section{Miniabrigo $\mathrm{ABC}$, usado no plano de manejo das unidades de conservação do trecho sul do rodoanel Mario Covas (SP-21)}

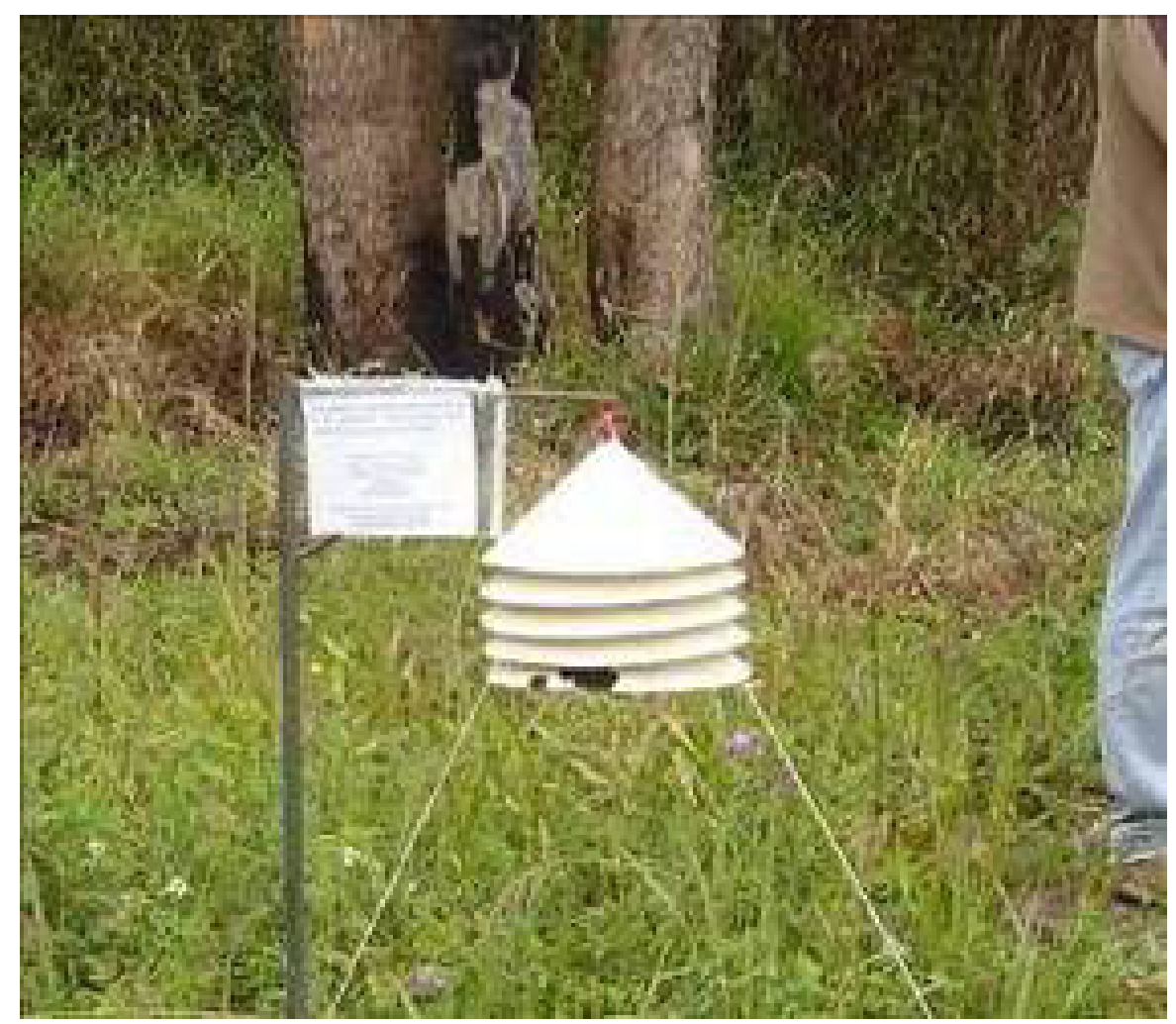

Posteriormente, também buscando uma outra alternativa de miniabrigo, Serafini Júnior (2010) desenvolveu um novo modelo destinado aos trabalhos de campo planejados à sua tese, onde os termo-higrômetros também pudessem ficar instalados e protegidos da ação direta do tempo (vento, chuva e sol), além de possibilitar o fácil transporte até a área de estudo. Esse novo modelo de miniabrigo foi nomeado de Quatro Águas (4AS).

Em linhas gerais, o miniabrigo 4AS é composto por quatro faces fechadas por venezianas e uma cobertura de madeira de quatro águas que também delimitam um ambiente interno de ventilação passiva, onde o termo-higrômetro é alojado. A instalação desse modelo é semelhante ao miniabrigo TAR, sustentado por uma haste de madeira e fixado por fitas ou cordões (Figura 3). 


\section{Figura 3}

\section{Miniabrigo 4AS, usado nos trabalhos de campo no Parque Estadual Veredas do Peruaçu (MG), entre 2009 e 2010}

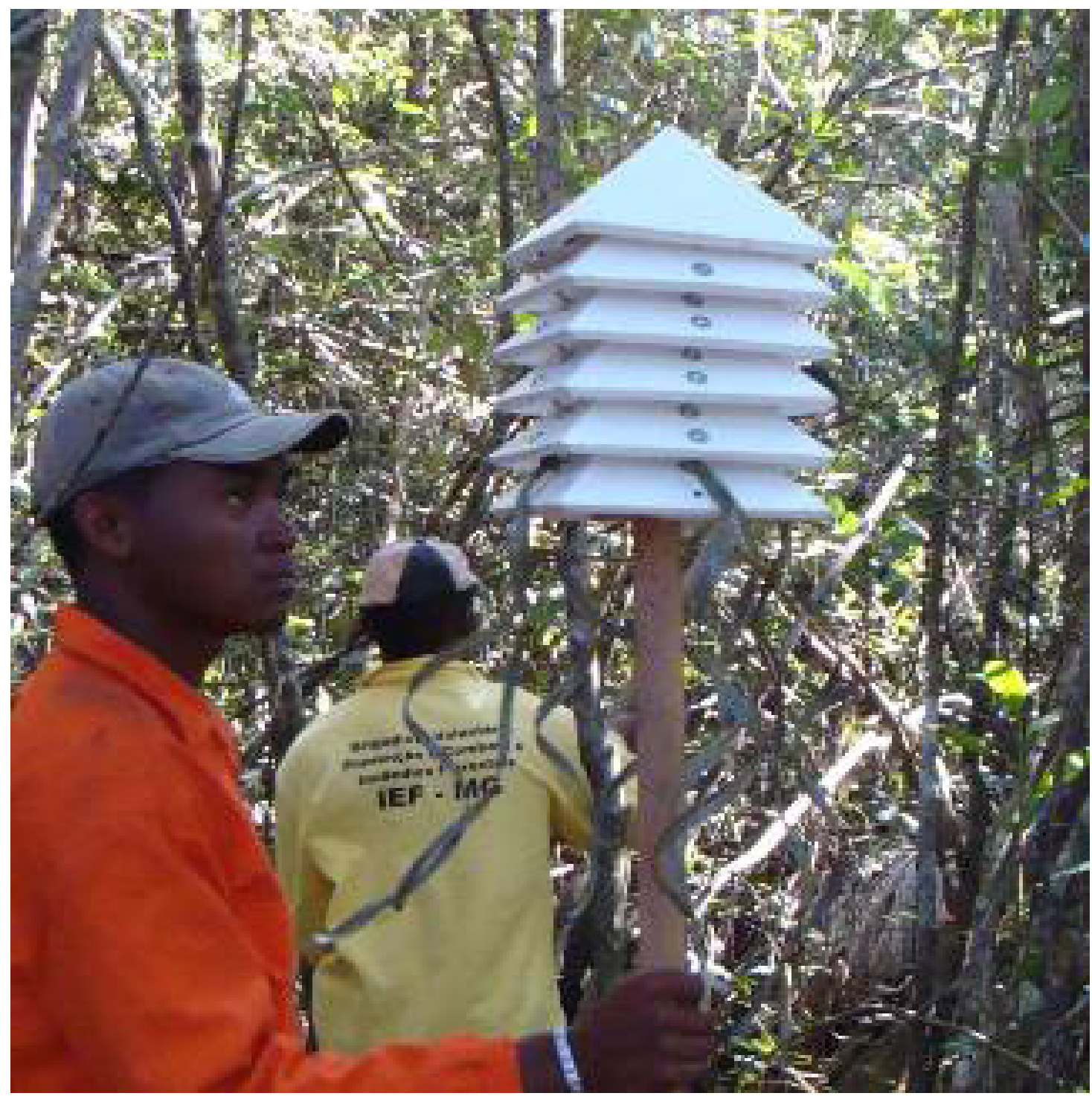

\section{Metodologia}

Para este trabalho, usaram-se quatro termo-higrômetros (modelo HT-500) para armazenar com alta precisão dados de temperatura $\left({ }^{\circ} \mathrm{C}\right)$ e umidade relativa do ar $(\%) .{ }^{4}$ Segundo dados divulgados pelo próprio fabricante, a acurácia ${ }^{5}$ térmica desse modelo é de $0,5^{\circ} \mathrm{C}$ para mais ou para menos.

Para dar início aos trabalhos, foi necessário calibrar esses equipamentos, para garantir que qualquer variação térmica registrada se devesse unicamente às características atmosféricas, independentemente do erro instrumental próprio de cada termo-higrômetro ou do modelo de miniabrigo utilizado. ${ }^{6}$

4 Neste artigo, usam-se apenas os dados de temperatura $\left({ }^{\circ} \mathrm{C}\right)$.

5 Acurácia é a precisão de uma medida ou de um instrumento.

6 A EMA não foi calibrada porque esse é um procedimento exclusivo do fabricante. 
A saber, a calibração é um procedimento de tratamento dos dados registrados pelos termo-higrômetros durante um período de tempo determinado, que possibilita o ajuste e a correção de eventuais diferenças associadas às características próprias de cada sensor (acurácia).

Após a calibração, foi feita a montagem de três grupos distintos de miniabrigos, compostos pelos modelos TAR, ABC e 4AS, com o objetivo de comparar os dados ali registrados com os da Estação Meteorológica Automática (EMA), localizada no mesmo ambiente (Figura 4).

\section{Figura 4}

Planta baixa da área da Estação Meteorológica Automática (EMA), onde os miniabrigos avaliados foram instalados entre 2010 e 2011.

(A) Miniabrigo 4AS, (B) Miniabrigo TAR e (C) Miniabrigo ABC

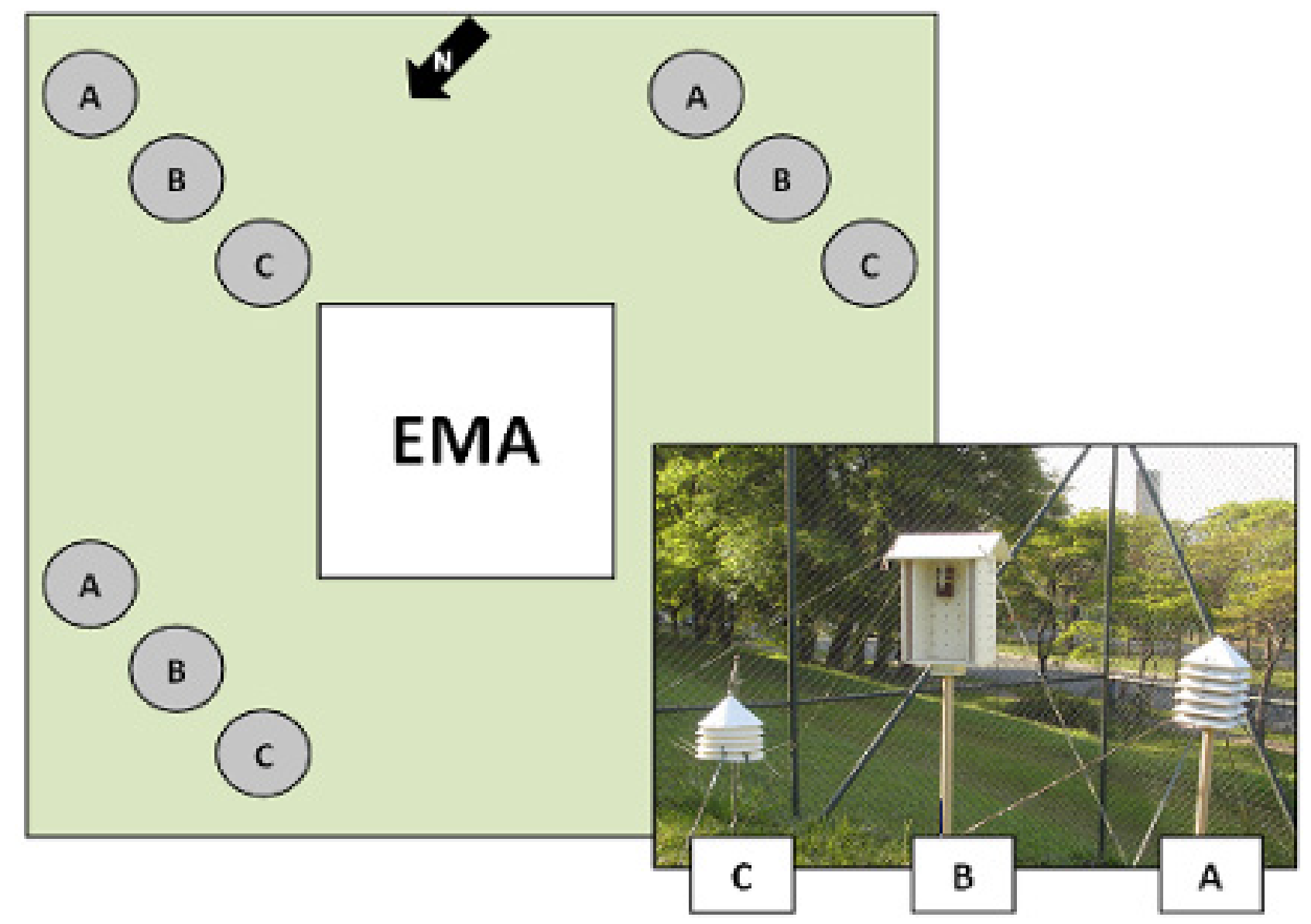

Para a análise do desempenho desses miniabrigos em relação à EMA, entendeu-se como necessário os dados de cada uma das estações do ano, a fim se mensurar essas comparações no contexto sazonal, através do cálculo da média aritmética. Para isto, as estações do ano em que os miniabrigos foram amostrados são da primavera de 2010 ao inverno de 2011 (Tabela 1). ${ }^{7}$

Após a análise sazonal, realizou-se uma segunda comparação dos dados registrados simultaneamente na EMA, vinculados aos períodos diurnos (das 6 h00 às 18h00) e noturnos (das $18 \mathrm{~h} 01$ às $5 \mathrm{~h} 59$ ).

7 Todos os dados registrados para esta publicação estão disponíveis para consulta no Laboratório de Climatologia e Biogeografia do Departamento de Geografia da Universidade de São Paulo. 


\section{Tabela 1}

Períodos de amostragem de temperatura para este trabalho

\begin{tabular}{c|c|c}
\hline CALIBRAÇÃO & PERÍODO DE AMOSTRAGEM & ESTAÇÃO DO ANO \\
\hline $18 / 10 / 2010$ & $23 / 10 / 2010$ a $20 / 11 / 2010$ & Primavera \\
\hline $20 / 01 / 2011$ & $23 / 01 / 2011$ a $20 / 02 / 2011$ & Verão \\
\hline $20 / 04 / 2011$ & $23 / 04 / 2011$ a $20 / 05 / 2011$ & Outono \\
\hline $20 / 07 / 2011$ & $23 / 07 / 2011$ a $20 / 08 / 2011$ & Inverno \\
\hline
\end{tabular}

Para a avaliação do desempenho dos miniabrigos na escala sazonal e diuturna, adotou-se como referência a amplitude térmica, que corresponde à extensão da amplidão entre as temperaturas média mínima e média máxima, registradas pelos termo-higrômetro instalados em cada miniabrigos e a EMA, simultaneamente.

Assim, a referência das menores amplitudes térmicas associadas a cada modelo de miniabrigo são indicativas da melhor eficiência de cada um deles no período correspondente. Isso demonstra que seu ambiente interno condiciona situação mais próxima ou semelhante ao desempenho da própria EMA, que possui certificado de calibração eletrônica emitido pelo próprio fabricante. Ou seja: pequena amplitude térmica = mais eficiência.

A seguir são apresentadas as análises de desempenho desses miniabrigos, a partir das temperaturas médias registradas simultaneamente entre eles e a EMA, referentes aos períodos de amostragem de verão, outono, inverno e primavera. Posteriormente outra análise é apresentada em relação aos períodos diurnos (das 6 h00 às 18h00) e noturnos (das 18h0l às 5h59) para o mesmo período sazonal.

\section{Análise sazonal}

No verão, o miniabrigo 4AS apresentou a menor amplitude térmica $\left(0,15^{\circ} \mathrm{C}\right)$ dentre todos os demais, que apresentaram amplitudes de $0,3^{\circ} \mathrm{C}(\mathrm{ABC})$ e $0,25^{\circ} \mathrm{C}(\mathrm{TAR})$ em relação á temperatura média registrada na $\mathrm{EMA}\left(23,8^{\circ} \mathrm{C}\right)$, no mesmo período.

No outono, os dados registrados no miniabrigo 4AS foram iguais aos da EMA (amplitude zero, portanto), enquanto que os miniabrigos $A B C$ e TAR condicionaram temperaturas mais elevadas e, consequentemente, amplitudes térmicas maiores $\left(0,3{ }^{\circ} \mathrm{C}\right.$ e $0,25{ }^{\circ} \mathrm{C}$, respectivamente).

No inverno, o miniabrigo TAR apresentou o melhor desempenho a partir da referência da amplitude térmica em relação à EMA $\left(0,2^{\circ} \mathrm{C}\right)$, enquanto que o $4 \mathrm{AS}$ e o TAR apresentaram amplitudes de $0,35^{\circ} \mathrm{C}$ e $0,40^{\circ} \mathrm{C}$, respectivamente, no mesmo período 
Na primavera, os resultados obtidos apontaram que os miniabrigos $A B C$ e TAR apresentaram amplitudes térmicas de $0,05{ }^{\circ} \mathrm{C}$ em relação à EMA, representando 0 melhor desempenho no período amostrado, enquanto que o resultado do 4AS foi o mais elevado dentre todos, com uma amplitude térmica de $0,1^{\circ} \mathrm{C}$ nesse período de amostragem.

Na Figura 5, são apresentados os gráficos demonstrativos das amplitudes térmicas calculadas para cada miniabrigo meteorológico, em que a linha vermelha é indicativa da condição exclusiva da EMA, ou seja, quanto mais próxima desta linha, melhor foi o desempenho do miniabrigo avaliados.

\section{Figura 5}

Amplitudes térmicas e temperaturas médias $\left({ }^{\circ} \mathrm{C}\right)$ registradas durante os períodos amostrais sazonais (verão, outono, inverno e primavera)

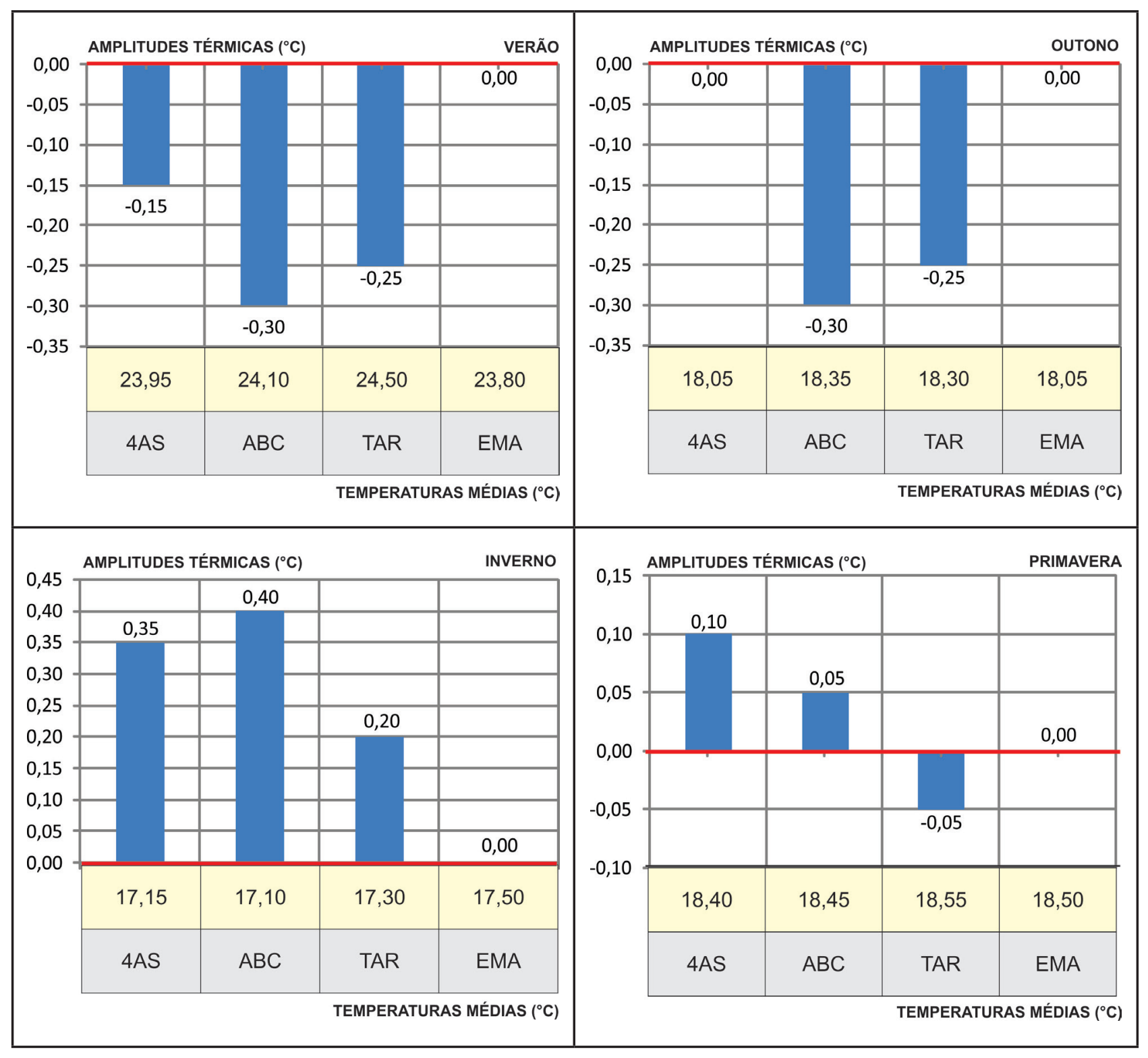


Portanto, tomando-se como referência as temperaturas médias registradas nos três modelos de miniabrigos e na EMA, para que fosse possível mensurar seus desempenhos, verificou-se que:

- No período do verão, o miniabrigo modelo 4AS apresentou o melhor desempenho dentre todos os demais, com uma amplitude térmica de $0,15^{\circ} \mathrm{C}$ em relação à EMA;

- No período de outono, o modelo 4AS também apresentou o melhor desempenho de todos, porque a amplitude térmica em relação à EMA foi zero.

- No período de inverno, o melhor desempenho foi do miniabrigo TAR, porque teve um amplitude térmica de $0,2^{\circ} \mathrm{C}$ em relação à EMA, entre todos os demais; e,

- No período da primavera, os miniabrigos modelos ABC e TAR apresentaram o melhor desempenho, quando registraram uma amplitude térmica de $0,05^{\circ} \mathrm{C}$ em relação à EMA.

\section{Análise diurna e noturna}

No período diurno do verão, a menor amplitude térmica ocorreu entre o miniabrigo 4AS e a EMA $\left(0,1^{\circ} \mathrm{C}\right)$, enquanto que nos demais, essas amplitudes foram bem superiores $\left(0,4^{\circ} \mathrm{C}\right.$ em relação ao $\mathrm{ABC})$ e $\left(0,3^{\circ} \mathrm{C}\right.$ em relação ao $\left.\mathrm{TAR}\right)$. No período noturno, a amplitude térmica foi homogênea em todos os miniabrigos em relação à EMA $\left(0,2^{\circ} \mathrm{C}\right)$.

No outono, o miniabrigo 4AS também condicionou a menor amplitude térmica em relação à EMA $\left(0,1^{\circ} \mathrm{C}\right)$ durante o dia, enquanto que no TAR e no $A B C$, as amplitudes diurnas foram de $0,6{ }^{\circ} \mathrm{C}$ e $0,8{ }^{\circ} \mathrm{C}$ em relação á EMA, respectivamente para o mesmo período. Durante a noite, as menores diferenças térmicas foram registradas nos miniabrigos $4 \mathrm{AS}$ e TAR (ambas com $0,1^{\circ} \mathrm{C}$ em relação á EMA), enquanto que em relação ao $A B C$, essa amplitude foi de $0,2^{\circ} \mathrm{C}$.

No período diurno do inverno, a menor amplitude térmica em relação à EMA está associada aos miniabrigos $4 \mathrm{AS}\left(0,1^{\circ} \mathrm{C}\right)$ e $\mathrm{ABC}$, enquanto que em relação ao $\mathrm{TAR}$, essa amplitude duplicou $\left(0,2^{\circ} \mathrm{C}\right)$. À noite, as menores amplitudes estiveram associadas aos miniabrigos $4 \mathrm{AS}$ e TAR (ambas com $0,6^{\circ} \mathrm{C}$ em relação á EMA), e de $0,9^{\circ} \mathrm{C}$ entre o miniabrigo $\mathrm{ABC}$ e essa mesma EMA.

Na primavera, as menores amplitudes térmicas diurnas estão associadas aos miniabrigos $4 \mathrm{AS}$ e $\mathrm{ABC}\left(0,1^{\circ} \mathrm{C}\right.$ em relação à EMA), enquanto para o TAR, essa amplitude foi de $0,2^{\circ} \mathrm{C}$ (o dobro). Durante a noite, foram constatadas as menores amplitudes térmicas no $4 \mathrm{AS}$ e o no TAR em relação à EMA $\left(0,1^{\circ} \mathrm{C}\right)$ e $0,2^{\circ} \mathrm{C}$ entre o miniabrigo $\mathrm{ABC}$ e a EMA.

Na Figura 6, são apresentados os gráficos demonstrativos das amplitudes térmicas descriminadas para os períodos diurnos e noturnos calculadas para cada miniabrigo meteorológico. Nos gráficos apresentados nesta figura, a linha vermelha é indicativa da condição exclusiva da EMA, ou seja, quanto mais próxima dela, melhor foi o desempenho do miniabrigo avaliados. 


\section{Figura 6}

\section{Amplitudes térmicas e temperaturas médias $\left({ }^{\circ} \mathrm{C}\right)$ registradas nos}

períodos diurno e noturno durante os períodos amostrais sazonais (verão, outono, inverno e primavera)

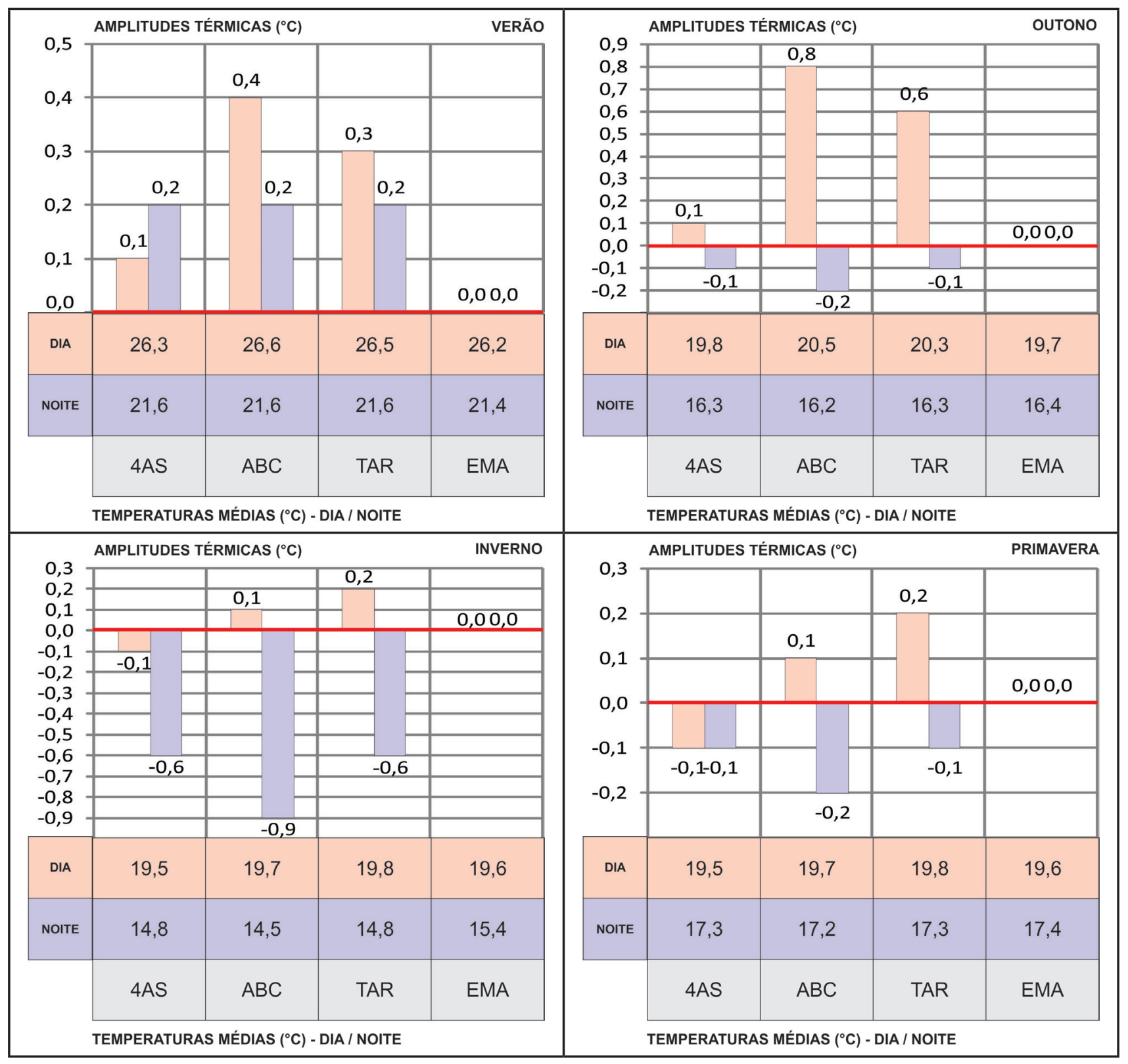

A partir das amplitudes térmicas aqui apresentadas e da acurácia dos data loggers indicada pelo fabricante $\left(+/-0,5^{\circ} \mathrm{C}\right)$, foi possível verificar que todos os miniabrigos avaliados apresentaram uma eficiência bastante satisfatória em 80\% de todo o período amostrado.

Ressalva deve ser dada ao período diurno do outono para os miniabrigos $\mathrm{ABC}$ e TAR, quando foram registradas amplitudes térmicas superiores à referida acurácia (0,5 ${ }^{\circ} \mathrm{C}$ ). Caso semelhante também foi constatado no período de inverno para todos os miniabrigos aqui avaliados (Figura 7). 


\section{Figura 7}

Amplitudes térmicas médias $\left({ }^{\circ} \mathrm{C}\right)$ registradas nos miniabrigos $4 \mathrm{AS}, \mathrm{ABC}, \mathrm{TAR}$ em relação à EMA (em valores absolutos), nos períodos diurno e noturno considerados, entre 2010 e 2011

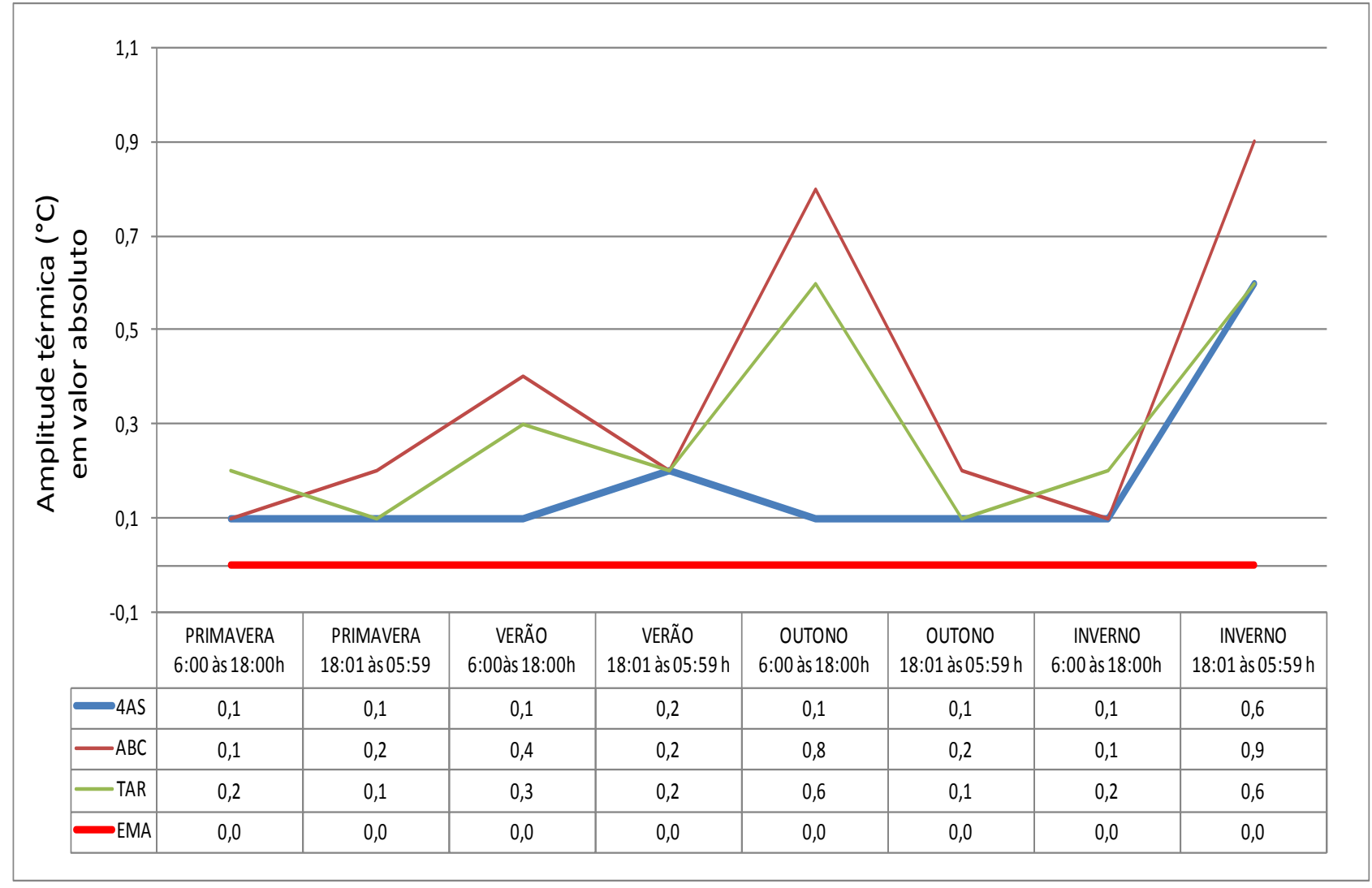

Associando as amplitudes térmicas ao desempenho de cada miniabrigo avaliado, verificou-se também que o modelo 4AS proporcionou a menor amplitude térmica em relação à EMA durante os períodos de primavera, verão, outono e inverno, com uma variação média de $0,15^{\circ} \mathrm{C}$, seguido pelos modelos $\operatorname{TAR}\left(0,19^{\circ} \mathrm{C}\right)$ e $\operatorname{ABC}\left(0,26^{\circ} \mathrm{C}\right)$.

Em relação ao período diurno e ao período noturno, foi constatado que o modelo 4AS também apresentou a melhor eficiência referenciada pela amplitude térmica em relação aos dados registrados na EMA.

A partir da constatação dos períodos em que as amplitudes térmicas calculadas variaram entorno do erro instrumental estabelecido pelo fabricante, foi elaborada a Tabela 2, com indicação semafórica simplificada (apenas cores verde e vermelha), permitindo inferir que o miniabrigo modelo 4AS, dentre todos demais, apresentou as condições mais favoráveis à estabilização das temperaturas ambientes a serem registradas por termo-higrômetros, frente às variações sazonais e diuturnas. 


\section{Tabela 2}

Indicação semafórica dos períodos adequados para uso de cada miniabrigo

\begin{tabular}{|c|c|c|c|c|}
\hline & PERÍODO & 4AS & $\mathrm{ABC}$ & TAR \\
\hline \multirow{2}{*}{ VERÃO } & das $06: 00$ às $18: 00 \mathrm{~h}$ & 0,10 & 0,40 & 0,30 \\
\hline & das $18: 01$ às $05: 59 \mathrm{~h}$ & 0,20 & 0,20 & 0,20 \\
\hline \multirow{2}{*}{ OUTONO } & das $06: 00$ às $18: 00 \mathrm{~h}$ & 0,10 & 0,80 & 0,60 \\
\hline & das $06: 00$ às $18: 00 \mathrm{~h}$ & $-0,10$ & $-0,20$ & $-0,10$ \\
\hline \multirow{2}{*}{ INVERNO } & das $18: 01$ às $05: 59 \mathrm{~h}$ & $-0,10$ & 0,10 & 0,20 \\
\hline & das $18: 01$ às $05: 59 \mathrm{~h}$ & $-0,60$ & $-0,90$ & $-0,60$ \\
\hline \multirow{2}{*}{ PRIMAVERA } & das $06: 00$ às $18: 00 \mathrm{~h}$ & $-0,10$ & 0,10 & 0,20 \\
\hline & das $18: 01$ às $05: 59 \mathrm{~h}$ & $-0,10$ & $-0,20$ & $-0,10$ \\
\hline \multicolumn{5}{|l|}{ LEGENDA: } \\
\hline & \multicolumn{4}{|c|}{ Período mais adequado para o uso do miniabrigo } \\
\hline & \multicolumn{4}{|c|}{ Período menos adequado para o uso do miniabrigo } \\
\hline
\end{tabular}

\section{Considerações finais}

Os miniabrigos usdos no Departamento de Geografia da Universidade de São Paulo foram desenhados e construídos para criar uma estrutura portátil, de fácil instalação em campo e que protegesse os equipamentos da ação direta do tempo (chuva, sol, vento etc.), garantindo o registro e o armazenamento de dados do tempo meteorológico sem grandes alterações das condições atmosféricas encontradas no ambiente externo analisado.

Assim, considerando os dados obtidos durante todo o período de amostragem nos miniabrigos $4 \mathrm{AS}, \mathrm{ABC}$, TAR, observou-se o desempenho de cada um a partir das amplitudes térmicas calculadas em relação à EMA instalada no mesmo ambiente.

Constatou-se que o desempenho de cada miniabrigo está diretamente associado aos seguintes aspectos:

- material utilizado na construção (madeira e latão galvanizado);

- aspectos arquitetônicos de cada miniabrigo e

- seus espaços internos diferentes, que são condicionantes de trocas térmicas com o espaço externo em que está instalado por meio de uma maior ou menor ventilação passiva.

Embora os resultados apresentados indiquem um desempenho dos miniabrigos bastante satisfatório durante $80 \%$ de todo o período amostrado, cabe destacar que todos eles apresentaram bons resultados. Espera-se que este trabalho contribua não só para que continuem sendo utilizados, mas para que novos modelos sejam projetados e utilizados em diferentes pesquisas em Geografia. 


\section{Referências}

ARMANI, G. Análise topo e microclimática tridimensional em uma microbacia hidrográfica de clima tropical úmido de altitude. Tese (Doutorado em Geografia Física) - Faculdade de Filosofia, Letras e Ciências Humanas, Universidade de São Paulo, São Paulo, 2009. Disponível em: <http://www.teses.usp.br/teses/disponiveis/8/8135/tde-02022010145510/>. Acesso em: 25 mar. 2012.

ARMANI, G.; GALVANI, E. Avaliação do desempenho de um abrigo meteorológico de baixo custo. Revista Brasileira de Agrometeorologia, Santa Maria, v. 14, p. 116-122, 2006.

AZEVEDO, T. R.; TARIFA, J. R. Miniabrigo meteorológico aspirado do Laboratório de Climatologia e Biogeografia e seu uso no estudo geográfico do clima. GEOUSP - Espaço e Tempo, São Paulo v. 10, p. 165-174, 2001.

INSTITUTO BRASILEIRO DO MEIO AMBIENTE E DOS RECURSOS NATURAIS RENOVÁVEIS - Ibama. Plano de Manejo do Parque Nacional Cavernas do Peruaçu. Brasilia: Ibama, 2005.

SERAFINI JÚNIOR, S. O microclima de uma vereda degradada: estudo de caso no Parque Estadual Veredas do Peruaçu-MG. Tese (Doutorado em Geografia Física) - Faculdade de Filosofia, Letras e Ciências Humanas, Universidade de São Paulo, São Paulo, 2010. Disponível em: <http://www.teses.usp.br/teses/disponiveis/8/8135/tde-08102010110002/>. Acesso em: 10 fev. 2012. 